\title{
An update on distribution models for Rhipicephalus microplus in West Africa
}

\author{
Eva M. De Clercq ${ }^{1}$, Agustin Estrada-Peña ${ }^{2}$, Safiou Adehan ${ }^{3}$, Maxime Madder $^{4}$, Sophie O. \\ Vanwambeke ${ }^{1}$ \\ ${ }^{1}$ Georges Lemaitre Institute for Earth and Climate Research, Université Catholique de Louvain, Louvain-la- \\ Neuve, Belgium; ${ }^{2}$ Unidad de Parasitología, Facultad de Veterinaria, Zaragoza, Spain; ${ }^{3}$ Ministère de l'Agriculture \\ de l'Elevage et de la Pêche/CeRPA-OP/Service Recherche Developpement, Porto Novo, Benin; ${ }^{4}$ Vector Biology \\ Unit, Institute of Tropical Medicine, Antwerp, Belgium
}

\begin{abstract}
The cattle tick, Rhipicephalus microplus, which reached the West African region approximately 8 years ago, has established viable populations in Côte d'Ivoire and Benin and spread rapidly from the assumed points of introduction. However, existing maps of its distribution range do not agree on the areas at risk, most probably due to suboptimal modelling approaches. Therefore, we undertook a re-investigation of the potential distribution range based on a high-quality dataset from West Africa that includes information on 104 farms located all over Benin. Focussing on climate suitability and applying advanced modelling, a subset of representative and uncorrelated climate variables was selected and fed into Maxent software to obtain an estimate of climate suitability for West Africa. The resulting map was validated using an independent dataset of 13 farms along the apparent distribution edge. The entire southern part of West Africa (covering southern Nigeria, Benin, Togo and Ghana) features high climate suitability for $R$. microplus. All of Côte d'Ivoire is inside the distribution range of this tick and the southern rim of Burkina Faso is expected to be suitable for the establishment of $R$. microplus populations. The validation of the distribution, dated one year after the initial field visit, confirmed the predicted distribution range, although a small number of individuals of R. microplus were found north of the predicted limit. These low numbers might indicate that the climate is not suitable for the establishment of a viable tick population. An alternative explanation is the recent introduction by nomadic cattle herds passing through this location. In this region of the world, it is quite common for cattle owners to lead their livestock over distances of more than $500 \mathrm{~km}$ in search of food and water.
\end{abstract}

Keywords: Rhipicephalus microplus, invasive species, species distribution models, climate suitability, Maxent, West Africa.

\section{Introduction}

Rhipicephalus microplus, a tick adapted to cattle, reached West Africa approximately 8 years ago (Madder et al., 2007) and has now established viable populations in Côte d'Ivoire and Benin (Madder et al., 2011, 2012). A country-wide survey in Benin has shown that it has since then spread rapidly from the assumed point of introduction in the town of Kpinnou in the South of Benin (De Clercq et al., 2012). After being introduced in Benin on imported cattle from Brazil, R. microplus has rapidly replaced the local blue tick. $R$. microplus is an effective, invasive species that is very resistant to acaricide pesti-

Corresponding author:

Eva M. De Clercq

Georges Lemaître Institute for Earth and Climate Research, Université Catholique de Louvain, Place Louis Pasteur 3

1348 Louvain-la-Neuve, Belgium

Tel. + 321047 9040; Fax +32 10472877

E-mail: eva.declercq@uclouvain.be cides, and it also spends a large part of its monotropic lifecycle attached to its ungulate host, so long-distance cattle movements can transport engorged female ticks throughout the tropical belt (Barré and Uilenberg, 2010). Moreover, this tick species is a good vector for both Babesia and Anaplasma pathogens, and its presence on cattle also causes weight loss due to blood-feeding, diminished milk production and increased mortality (Corrier et al., 1979; Guerrero et al., 2007; da Rocha et al., 2011; Madder et al., 2011; Da Silva et al., 2013). In addition, its presence in West Africa has resulted in increased and inappropriate acaricide use.

A major part of the human populations in West Africa has limited access to protein sources, and the spread of $R$. microplus might jeopardise efforts to improve protein intake, e.g. by milk consumption. Because of the threat that this species poses for animal production, it is the topic of several research projects being undertaken in the West African region (TickRisk, 2012; WecaTic, 2012). In order to design an efficient control strategy, there is a need for tar- 
geted surveillance. However, since resources available for monitoring are scarce, surveyed locations should be limited to areas over which this species could be expected to spread over the next few years (Obsomer et al., 2012). Efforts should thus be targeted at areas where a given species can establish itself, meaning that it can not only survive here, but it can maintain viable populations and reproduce over several years. This region is referred to as the potential distribution range, and is determined by environmental factors and can be described by species distribution models (SDMs), where occurrence records are analysed using a set of environmental variables, describing the biological requirements of the species, such as climate, topography or land cover (Hirzel et al., 2006). The distribution range for R. microplus in West Africa has been estimated and is published in the existing literature, but the area at risk of invasion by this species varies considerably from study to study (Cumming, 2000; Sutherst and Bourne, 2009). Early studies indicated that the environment in West Africa is not suitable for the survival of this tick (Cumming, 2000), while more recent papers instead claim that the West African region is highly favourable, in fact even more favourable than South Africa, the region of the original distribution of $R$. microplus (Sutherst and Bourne, 2009). Not only do these papers offer contradictory conclusions, but there are also concerns about the data on the occurrence of the tick and the methodology used to fit the models. The paper by Cumming (2000), for example, used the observed occurrences of $R$. microplus, which was at that time restricted to Southern Africa, to predict the potential distribution range in West Africa, and thus the SDM are extrapolated over a very large area. The results should be treated with caution as conventional SDM approaches, such as logistic regression used by Cumming (2000), are not particularly suitable for extrapolation. The extrapolation ability of a model is further decreased when many (correlated) variables are used to fit the model. The CLIMEX approach used by Sutherst and Bourne (2009) was designed to be extrapolated over large areas, but the implemented "four-point" algorithm is quite simplistic and does not take seasonality into account. They used the tick occurrence data collected by Cumming and completed this database with a small number of $R$. microplus records in West Africa.

Over the last decade, more complex and robust SDMs, allowing the prediction of the distribution range outside of the original occurrence area, have become available. The objective of this paper is to improve existing maps on the potential distribution range of R. microplus in West Africa. The main goal is to explore the limits of the potential distribution by extrapolating the environmental requirements of this tick. The host will not be taken into account in this study, nor will we address the presence of geographical barriers. We will focus on climate, as this is the main limiting factor. The potential distribution range is thus estimated by climate suitability. Two climatic aspects, temperature and water saturation deficit, determine the suitability for ticks. Water saturation deficit is the combined effect of temperature and relative humidity. Climate suitability for R. microplus in West Africa was estimated using (i) a high-quality dataset with occurrence records from the West African region; and (ii) robust SDM models. A clear indication of the areas at risk of invasion will be an important first step towards an effective control strategy for $R$. microplus in West Africa.

\section{Material and methods}

\section{Study area}

Tick data were available for Benin, but the study focused on the entire region of West Africa as it was considered to be at risk of invasion by $R$. microplus (Fig. 1a). This region covers four broad ecozones (FRA, 2000) described by the kind of forest that would be the natural vegetation given the local climate and geology. Seen from North to South these are: (i) tropical shrub land (TBSh); (ii) tropical dry forest (TAwb); (iii) tropical moist deciduous forest (TAwa); and (iv) tropical rainforest (TAr).

\section{Tick data}

Observed presence or absence of the cattle tick was collected in 104 randomly selected farms throughout Benin at the end of the rainy season in 2011 (De Clercq et al., 2012). Two animals with visible tick infestation were sampled at each location. The cattle tick was found in nearly all locations in the southern half of the country, while it is largely absent from the northern half of the country (Fig. 1b). More information on the selection of farms, the tick sampling protocol and the tick identification process can be found in De Clercq et al. (2012). The sampling took place at the end of the rainy season, and very high amounts of $R$. microplus ticks were found on infested animals. The observed absence points were considered to be reliable. 
(a)

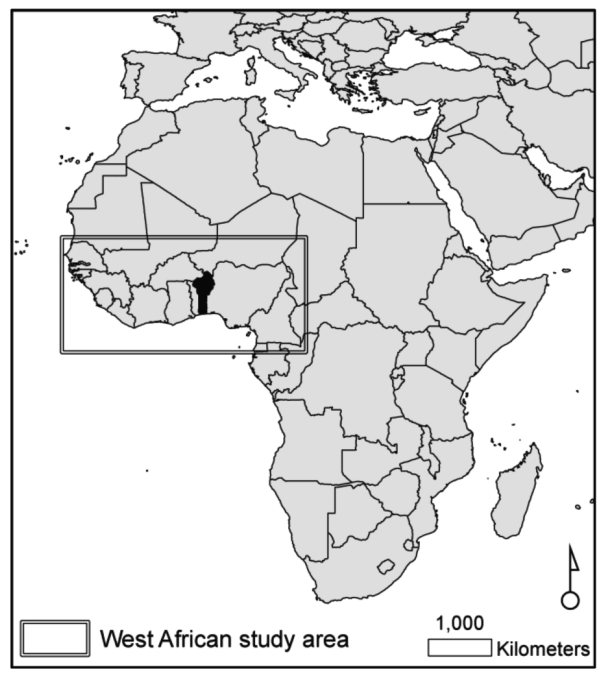

(b)

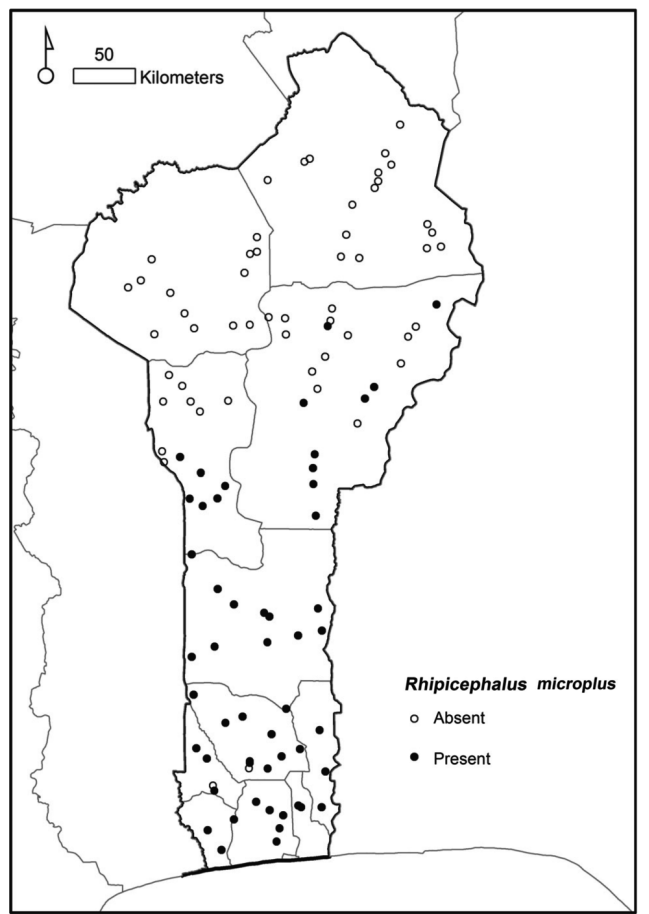

Fig. 1. The geographical location of Benin in West Africa (a) with the sampling points for R. microplus (b).

\section{Climate data}

The study is based on the most widely used climate dataset for species distribution modelling, i.e. the Worldclim dataset, which contains 19 bioclimatic variables (Hijmans et al., 2005). It contains the most important variables influencing tick survival and development at a spatial resolution of $1 \mathrm{~km}$ (Table 1 ). It contains monthly values for temperature and precipitation, which can be used as a proxy for water saturation deficit in a long-term global dataset of measures made at weather stations between 1950 and 1990. The influence of global warming has caused temperatures worldwide to be currently between 0.2 and $0.3{ }^{\circ} \mathrm{C}$ warmer than the $1961-1990$ average (Hulme et al., 2001, Sarr, 2012). Concerning rainfall, however, the West African climate has been very variable. The period between 1930 and 1960 was wetter than normal, 1970-1980 was characterised by droughts and rainfall in the 1990s was average. Since the mid-1990s a wetting trend is observed (Sarr, 2012). In short, the range of inter-annual variability when averaging over 30 years is considered larger than the change in precipitation since the 1990s (Hulme et al., 2001). Although the Worldclim data might seem outdated, we considered this dataset a good-enough descriptor of current West African climate. The decision not to use land cover information was based on the fact that this variable is subject to human impact, and changes rapidly in developing countries (Hulme et al., 2001). The main asset of the Worldclim dataset is that it is readily available for modelling purposes without any form of computer-intensive pre-processing.

Since many of these Worldclim variables are assumed to be highly correlated, a principal component analysis (PCA) was performed in order to assist selection of useful variables. During PCA, the original correlated variables are combined to create new variables. This is done in such a manner that the newly created variables are orthogonal, meaning that they are no longer intercorrelated. The combined variables are referred to as principal components (PCs), which can be derived using the following formula:

$$
\begin{gathered}
\text { PC } 1=\text { loading } 1 \times \text { variable } 1+\text { loading } 2 \\
\text { x variable } 2+\ldots \ldots
\end{gathered}
$$

Results were examined on the biplot, a scatterplot where the X-and Y-axis are the first and second PCs. Presence/absence records were also plotted on the biplot providing a graph that allows analysis of the correlation between the original variables. The variable selection for the potential distribution modelling of $R$. microplus was based on biological grounds (Rodda et al., 2011). The PCA analysis was performed to gain understanding of the correlation between the variables in the Worldclim dataset and to assure that the variables selected were uncorrelated. 


\section{Geographical information system (GIS)}

ArcGIS software (ESRI; Redlands, USA) was used to process spatial data, project the information onto a geographic coordinate system, resample all raster layers to $1 \mathrm{~km}$ resolution and prepare the maps (ESRI, 2009). Data layers of administrative boundaries and general topography were downloaded from the Map Maker Trust (Map Library, 2012).

\section{Climate suitability maps}

The goal of species' potential distribution modelling is to compute the probability of presence in locations not sampled. Generally, occurrence records are analysed using a set of environmental variables, characteristic for a species native range climate (Rodda et al., 2011). Once the relationships between environmental variables and the occurrence data are established, they can be used to project a presence of the tick into geographical space, and results can be extrapolated to predict the outcome at un-sampled locations. We used the maximum entropy (Maxent) approach, as implemented in the Maxent stand-alone programme (Phillips and Dudik, 2008), which is known to perform SDM with high accuracy (Elith et al., 2006). It produces a map with cell values varying between 0 and 1 , indicating how close the climate in each cell is to the optimal condition for the species in question: the higher the value, the higher the suitability. As a rule of thumb, sites with suitability higher than 0.5 predict presence, while sites with suitability lower than 0.5 indicate absence. The limit of the distribution is defined as the zone featuring a suitability value between 0.0 and 0.1 .

When reliable absence indications are not available, Maxent uses random background as absence (Elith et al., 2011). In this study, however, reliable absence data were available and the "background" points were selected from the locations visited where R. microplus was not found (Fig. 1). The data regarding presence and absence were randomly split in a calibration and a validation dataset. The model was fitted using $90 \%$ of the data, and the accuracy of the model was assessed on the remaining $10 \%$ of the data. This approach was iterated 10 times, resulting in 10 repetitions. The selected variables were fed to a Maxent model, using 10 repetitions and hinge features. Setting the features to "hinge" indicates that the model looks for piece-wise linear relations between a given Worldclim variable and the suitability for R. microplus. The relation between an environmental variable and suitability will have the aspect of a "broken stick". The model was projected for the entire region of West Africa. We used only a small number of variables in order to avoid "overfitting", which has been reported as a problem when a large set of predictors is used (Jiménez-Valverde et al., 2011). In the case of "overfitting", the model uses a large part of the available predictor variables and fits very close to the training data. These models often contain relations that have no biological foundation, resulting in models with poor generalisation potential (Elith et al., 2006; Jiménez-Valverde et al., 2011; Rodda et al., 2011).

\section{Model validation}

Since $R$. microplus is a species that invaded West Africa recently, it was all the more interesting to test the resulting climate suitability maps using an independent dataset. We set out a north-south gradient at the apparent distribution edge and revisited approximately the same locations in this area of the original survey of 2011 exactly one year later. Between 26 and 29 October 2012, 13 points were visited covering a $200 \mathrm{~km}$-long transect (Fig. 2). Each point was visited once and ticks were collected from two animals. The sampling method was identical to the one used in 2011 for the calibration dataset.

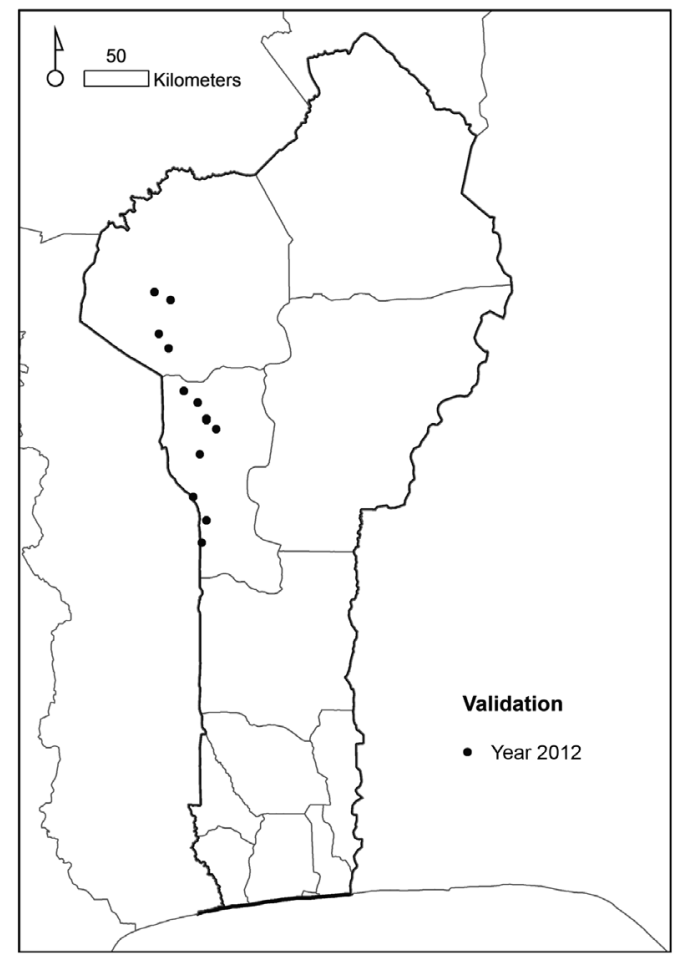

Fig. 2. The locations in Benin visited during the validation campaign in 2012. 


\section{Results}

\section{Climate data correlations}

The 19 bioclimatic variables showed a high correlation, the first two components explaining $86 \%$ of all variation. The loadings for the first and second PCs are described in Table 1, while Fig. 3 shows a scatterplot of the observations along the first two PCs. Each point in Fig. 3 represents a location that was sampled in 2011; the " $\mathrm{x}$ "-symbols indicate occurrences of R. microplus, while the "o"-symbols represent locations where $R$. microplus was not found. Nearly all $R$. microplus presences appear to be clustered along a gradient from the upper left to the lower right. R. microplus absences are situated in the right part of the graph. Red arrows indicate the relation of the original variables with the PCs. No clear clustering of variables could be observed, but there was a striking correlation between Bio_06, Bio_09 and Bio_17, as well as between Bio_07 and Bio_15. The first group of variables describes the temperature and precipitation in the driest period. The second group of variables describes the seasonal variation.

Both temperature and water availability are the main drivers of the tick life cycle, regulating the development and the mortality rates of the complete "offhost" life cycle. The following uncorrelated bioclimat- ic variables describe these climate requirements: (i) maximum temperature of the warmest month (Bio_05); (ii) annual precipitation (Bio_12); and (iii) precipitation during the driest quarter (Bio_17).

It was assumed that the surveyed area is well within the temperature limits of $R$. microplus, reported to be between $19-29^{\circ} \mathrm{C}$ (Joydhar et al., 2010).

\section{Climate suitability map}

The entire southern part of the study area features high climate suitability for $R$. microplus. This region covers the southern parts of Nigeria, Benin, Togo and Ghana. The entire country of Côte d'Ivoire falls inside the distribution range of this tick. Also the southern rim of Burkina Faso is expected to be suitable for sustaining $R$. microplus populations. This was consistent with the known distribution of $R$. microplus (Fig. 4). The standard deviation on this estimate over 10 repetitions was maximum 0.07 and this was considered low. The highest variability was observed along the limits of the distribution range. Another region of high variability was situated along the coast of Ghana and Côte d'Ivoire. The average test AUC for the replicate runs is 0.70 , and the standard deviation is 0.08 . The precipitation during the driest quarter (Bio_17) was the most important variable, contributing $94.2 \%$ to the model, while the maximum temperature of the

Table 1. The 19 bioclimatic variables in the Worldclim dataset and their correlation with the first and second principal component (PC).

\begin{tabular}{llcc}
\hline Code & & \multicolumn{1}{c}{ Description } & PC 1 \\
\hline BIO_01 & Annual mean temperature & -0.11 & 0.39 \\
BIO_02 & Mean diurnal range & 0.28 & -0.04 \\
BIO_03 & Isothermality & -0.24 & -0.23 \\
BIO_04 & Temperature seasonality & 0.24 & 0.25 \\
BIO_05 & Maximum temperature of the warmest month & 0.25 & 0.19 \\
BIO_06 & Minimum temperature of the coldest month & -0.29 & 0.01 \\
BIO_07 & Temperature annual range & 0.29 & 0.08 \\
BIO_08 & Mean temperature of the wettest quarter & -0.21 & 0.28 \\
BIO_09 & Mean temperature of the driest quarter & -0.27 & 0.03 \\
BIO_10 & Mean temperature of the warmest quarter & 0.10 & 0.41 \\
BIO_11 & Mean temperature of the coldest quarter & -0.15 & 0.35 \\
BIO_12 & Annual precipitation & -0.01 & -0.39 \\
BIO_13 & Precipitation of the wettest month & 0.24 & -0.00 \\
BIO_14 & Precipitation of the driest month & -0.26 & 0.06 \\
BIO_15 & Precipitation seasonality & 0.28 & -0.14 \\
BIO_16 & Precipitation of the wettest quarter & 0.26 & -0.29 \\
BIO_17 & Precipitation of the driest quarter & -0.23 & 0.01 \\
BIO_18 & Precipitation of the warmest quarter & 0.06 & -0.35 \\
BIO_19 & Precipitation of the coldest quarter & &
\end{tabular}


(A)

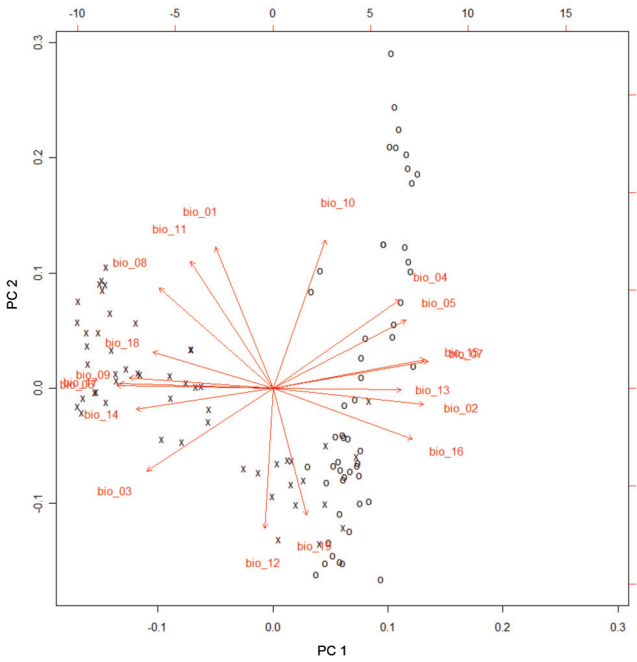

(B)

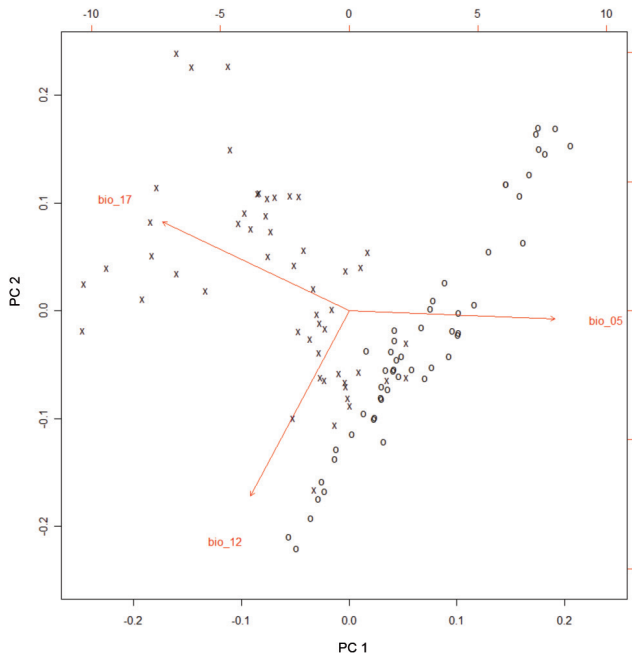

Fig. 3. Biplot showing the first two principal components (PCs) after principal component analysis (PCA). All points indicate locations sampled in 2011 with occurrences of R. microplus (x) and locations where R. microplus was not found (o). Graph (A) shows the result for all 19 bioclimatic variables, and graph (B) contains the subset of variables selected on biological grounds.

warmest month (Bio_05) and the annual precipitation (Bio_12) contributed $4.6 \%$ and $1.2 \%$, respectively.

\section{Model validation}

Fig. 5 shows the mapping of the result of the independent validation mission. In the areas with an unsuitable climate for the cattle tick (blue), no R. microplus ticks were found. In the southern part of the transect, the climate is more suitable and high numbers of this tick, ranging from 126 to 181 specimens per animal, were collected. In the transition zone, one or two R. microplus ticks were found. This could indicate that the tick has continued its spread northwards. The low numbers indicate that the climate is not suitable for the tick to establish a viable population. An alternative explanation is a recent introduction by transhumant cattle passing through this location.

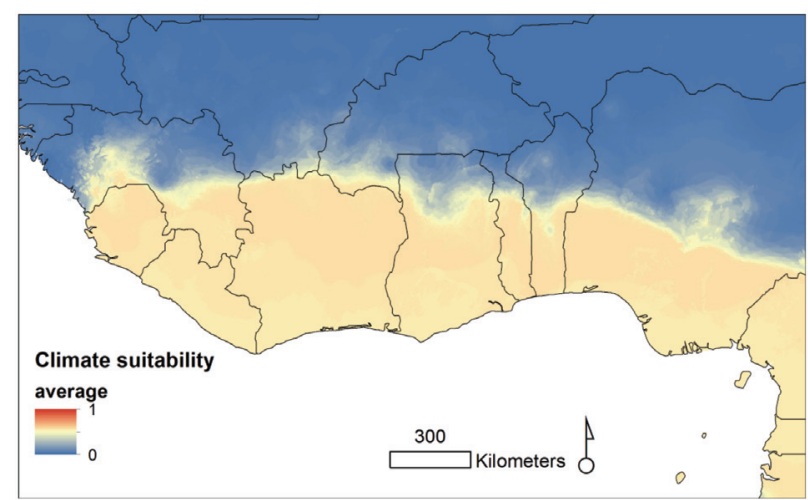

\section{Discussion}

After introduction of $R$. microplus in the south of Benin roughly a decade ago, this tick has spread and reached the northern departments of Benin. Predictions of the range over which this species could spread further indicate that while it has reached its full extent in Benin, the neighbouring countries are at risk of invasion. Indeed, the prevailing climate would allow this tick to spread to Togo and Ghana, going as far north as the southern rim of Burkina Faso. This area coincides more or less with the range previously predicted by CLIMEX (Sutherst and Bourne, 2009), which was developed after the establishment of R. microplus in Côte d'Ivoire was reported, the model was probably fine-tuned using this information.

One of the reasons the model by Cumming (2000) failed to predict the species range of $R$. microplus in West Africa is that 49 predictor variables were used,

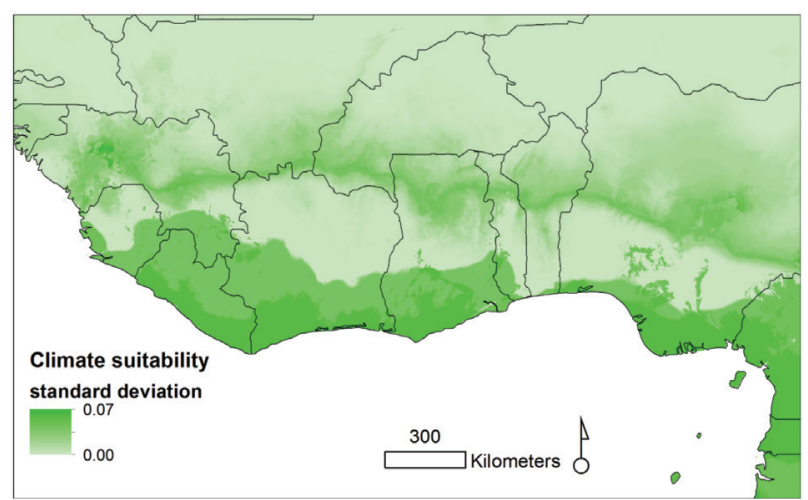

Fig. 4. Predicted climate suitability in sub-Saharan Africa for R. microplus. 


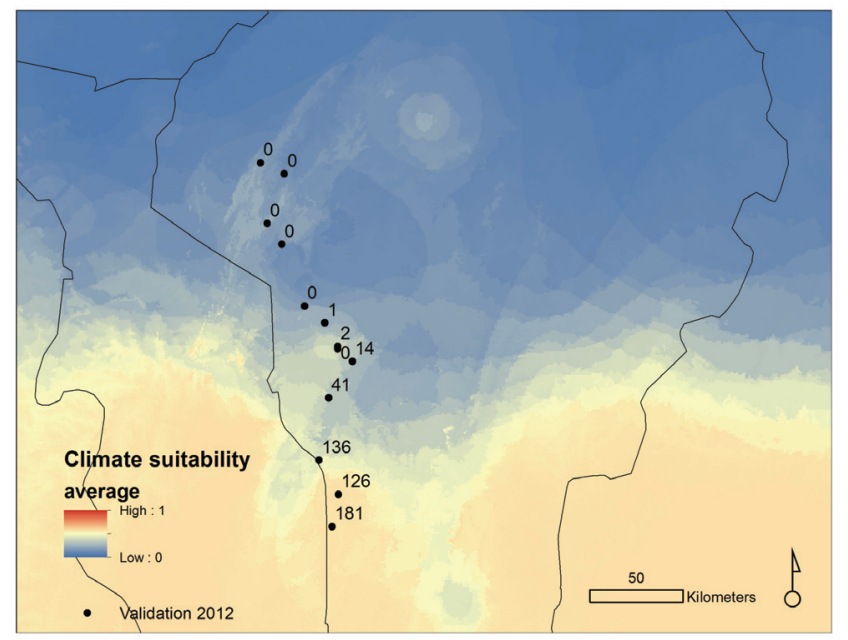

Fig. 5. Location of the sampled locations used for model validation in 2012 and predicted climate suitability in northern Benin. The number of adult ticks of the R. microplus species collected in 2012 at each sampling point.

which are highly correlated with each other. A variable selection procedure could have ruled out insignificant variables, but a larger problem is that using yearly aggregated variables (e.g. mean temperature) fail to record actual seasonal trends. Although the set of variables describes the variation of climate throughout the year by means of the mean monthly rainfall for each month (12 variables), as well as the mean, maximum and minimum temperatures for each month (three times 12 variables), the timing of dry seasons and temperature fluctuations is different throughout the continent, particularly above and below the equator. For example, March is the hottest month of the year in Burkina Faso, but this is not the case for Senegal or Tanzania (hottest in October), and not for Southern Africa (hottest in January). The bioclimatic variables in the Worldclim dataset mitigate this temporal shift in seasonality in different geographic regions, since they explicitly contain the values for, temperature and precipitation for respectively, the hottest, coolest, driest and wettest periods of the year.

\section{Conclusions}

Since nearly all West African countries are likely to import Brazilian cattle, an estimate of the distribution range over which this species could spread further is required to assess the threat this tick poses on animal production in the entire region of West Africa. As R. microplus is an exotic species in West Africa, it is far from certain that its current distribution has reached its full extent.
It is striking that two modelling approaches using different datasets, different predictor variables and a different modelling technique, agree to a large degree on the northern limit for R. microplus. Nonetheless, further monitoring of this invasive species will yield important information, not only from a veterinary point of view, but also for modelling of invasive species in general. Further longitudinal research and follow-up is needed to confirm this hypothesis.

\section{Acknowledgements}

This work was funded by the Belgian Science Policy Programs (Belspo, SR/00/144). E. M. De Clercq is Chargé de Recherche of the Fonds de la Recherche Scientifique (FNRS). We would like to express our thanks to the members of the steering committee of the TickRisk project, in particular Beth Purse, for their valuable input during the annual meetings. Finally, we would like to thank the anonymous reviewers for their constructive remarks.

\section{References}

Barré N, Uilenberg G, 2010. Spread of parasites transported with their hosts: case study of two species of cattle tick. Rev Sci Tech 29, 135-147, 149-160.

Corrier DE, Vizcaino O, Terry M, Betancourt A, Kuttler KL, Carson CA, Trevino G, Ristic M, 1979. Mortality, weight loss and anaemia in Bos taurus calves exposed to Boophilus microplus ticks in the tropics of Colombia. Trop Anim Health Prod 11, 215-221.

Cumming GS, 2000. Using habitat models to map diversity: Pan-African species richness of ticks (Acari: Ixodida). J Biogeogr 27, 425-440.

da Rocha CMBM, Leite RC, Bruhn FRP, Guimarães AM, Furlong J, 2011. Perceptions of milk producers from Divinópolis, Minas Gerais, regarding Rhipicephalus (Boophilus) microplus control. Rev Bras Parasitol Vet 20, 295 302.

Da Silva JB, Rangel CP, De Azevedo Baêta B, Da Fonseca AH, 2013. Influence of the physiological state on infestation by Rhipicephalus microplus in dairy cows. Ticks Tick Borne Dis 4, 52-56.

De Clercq EM, Vanwambeke S, Sungirai M, Adehan S, Lokossou R, Madder M, 2012. Geographic distribution of the invasive cattle tick Rhipicephalus microplus, a country-wide survey in Benin. Exp Appl Acarol 58, 441-452.

Elith J, Graham RPACH, Zimmermann NE, 2006. Novel methods improve prediction of species' distributions from occurrence data. Ecography 29, 129-151.

Elith J, Phillips SJ, Hastie T, Dudik M, Chee YE, Yates CY, 2011. A statistical explanation of MaxEnt for ecologists. Divers Distrib 17, 43-57. 
ESRI, 2009. ArcGIS Desktop: Release 9. Redlands, CA, USA, ESRI.

FRA, 2000. Global ecological zones. Available at: www.fao.org/geonetwork/srv/en/metadata.show?id=1255 (accessed on June 2013)

Guerrero FD, Bendele KG, Davey RB, George JE, 2007. Detection of Babesia bigemina infection in strains of Rhipicephalus (Boophilus) microplus collected from outbreaks in South Texas. Vet Parasitol 145, 156-163.

Hijmans RJ, Cameron SE, Parra JL, Jones PG, Jarvis A, 2005. Very high resolution interpolated climate surfaces for global land areas. Int J Climatol 25, 1965-1978.

Hirzel AH, Lay GL, Helfer V, Randin C, Guisan A, 2006. Evaluating the ability of habitat suitability models to predict species presences. Ecol Model 199, 142-152.

Hulme M, Doherty R, Ngara T, New M, Lister D, 2001. African climate change: 1900-2100. Clim Res 17, 145-168.

Jiménez-Valverde A, Peterson AT, Soberón J, Overton JM, Aragón P, Lobo JM, 2011. Use of niche models in invasive species risk assessments. Biol Invasions 13, 2785-2797.

Joydhar NB, Haq MS, Rahman MH, Sarkar AK, 2010. Laboratory studies on the life cylce of Boophilus microplus. Int J Bio Res 2, 13-18.

Madder M, Adehan S, De Deken R, Adehan R, Lokossou R, 2012. New foci of Rhipicephalus microplus in West Africa. Exp Appl Acarol 56, 385-390.

Madder M, Thys E, Achi L, Touré A, De Deken R, 2011. Rhipicephalus (Boophilus) microplus: a most successful invasive tick species in West-Africa. Exp Appl Acar 53, 139-145.
Madder M, Thys E, Geysen D, Baudoux C, Horak I, 2007. Boophilus microplus ticks found in West Africa. Exp Appl Acarol 43, 233-234.

Map Library, 2012. Available at: www.maplibrary.org (accessed on August 2013).

Obsomer V, Defourny P, Coosemans M, 2012. Predicted distribution of major malaria vectors belonging to the Anopheles dirus Complex in Asia: ecological niche and environmental influences. PLoS One 7, e50475.

Phillips SJ, Dudik M, 2008. Modeling of species distributions with Maxent: new extensions and a comprehensive evaluation. Ecography 31, 161-175.

Rodda GH, Jarnevich CS, Reed RN, 2011. Challenges in identifying sites climatically matched to the native ranges of animal invaders. PLoS One 6, e14670.

Sarr B, 2012. Present and future climate change in the semi-arid region of West Africa: a crucial input for practical adaptation in agriculture. Atmospheric Sci Lett 13, 108-112.

Sutherst RW, Bourne AS, 2009. Modelling non-equilibrium distributions of invasive species: a tale of two modelling paradigms. Biol Invasions 11, 1231-1237.

TickRisk, 2012. TickRisk: assessing ecological suitability for the spread of Rhipicephalus (Boophilus) microplus in West Africa. Available at: http://www.elic.ucl.ac.be/repomodx/elic/ index.php?id=232 (accessed on June 2013).

WecaTic, 2012. Assessment of emerging livestock ticks and tickborne disease threats and integrated control strategies in West and Central Africa. Available at: http://wecatic.over-blog.com/ (accessed on August 2013). 